\title{
Isolation of Nebulin from Rabbit Skeletal Muscle and Its Interaction with Actin
}

\author{
Ryo Chitose, Atsushi Watanabe, Masato Asano, Akira Hanashima, Kouhei Sasano, \\ Yulong Bao, Koscak Maruyama, and Sumiko Kimura
}

Department of Biology, Graduate School of Science, Chiba University, Chiba 263-8522, Japan

Correspondence should be addressed to Sumiko Kimura, sumiko@faculty.chiba-u.jp

Received 1 December 2009; Accepted 15 February 2010

Academic Editor: Henk L. M. Granzier

Copyright (C) 2010 Ryo Chitose et al. This is an open access article distributed under the Creative Commons Attribution License, which permits unrestricted use, distribution, and reproduction in any medium, provided the original work is properly cited.

\begin{abstract}
Nebulin is about $800 \mathrm{kDa}$ filamentous protein that binds the entire thin filament of vertebrate skeletal muscle sarcomeres. Nebulin cannot be isolated from muscle except in a completely denatured form by direct solubilization of myofibrils with SDS because nebulin is hardly soluble under salt conditions. In the present study, nebulin was solubilized by a salt solution containing $1 \mathrm{M}$ urea and purified by DEAE-Toyopearl column chromatography via $4 \mathrm{M}$ urea elution. Rotary-shadowed images of nebulin showed entangled knit-like particles, about $20 \mathrm{~nm}$ in diameter. The purified nebulin bound to actin filaments to form loose bundles. Nebulin was confirmed to bind actin, $\alpha$-actinin, $\beta$-actinin, and tropomodulin, but not troponin or tropomyosin. The data shows that full-length nebulin can be also obtained in a functional and presumably native form, verified by data from experiments using recombinant subfragments.
\end{abstract}

\section{Introduction}

Single molecules of nebulin (the precise molecular weight expressed in a certain tissue depends on the respective splice isoform; for a $773 \mathrm{kDa}$ species, see [1]) associate along the entire length of the thin filament of vertebrate skeletal muscle. Therefore, nebulin is thought to be a molecular ruler for determining length of $\sim 1 \mu \mathrm{m}$ [2-4]. To ascertain such potential of nebulin, its effect on the length of actin filaments should be examined in vitro. However, nebulin has not been successfully isolated from skeletal muscle due to difficulties in solubilization under salt conditions, although isolation of a completely denatured nebulin has been achieved by gel filtration of SDS-solubilized myofibrils [5].

Complete sequencing of human nebulin by Labeit and Kolmerer showed that a large portion $(97 \%)$ of nebulin comprises 185 repeats of approximately 35 amino acids with a central consensus sequence of SDXXYK [1].

In the sarcomere, the $\mathrm{N}$-terminal region of nebulin localizes at the pointed end of the thin filaments, central region along the thin filaments, and C-terminal region in the Z-line [2]. Each nebulin repeat (SDXXYK) can bind to actin [6-8], while nebulin repeats $1-3$ bind to tropomodulin [9], 163-170 bind to desmin [10], 185-SH3 domain bind to connectin (also called titin) [11], and the $\mathrm{SH} 3$ domain binds to myopalladin [12] and $\beta$-actinin (also called CapZ) [11].

In 2009, Yadavalli et al. reported that they extracted full-length nebulin from rabbit longissimus dorsi muscle using $0.45 \%$ sodium deoxycholate (DOC), and then purified it with an $\mathrm{NaCl}$ gradient in the presence of $0.45 \%$ DOC. However, the detailed preparation method has not been published yet [13].

In the present study, we describe the purification of nebulin from rabbit skeletal muscle. For solubilization, $1 \mathrm{M}$ urea was required, and during purification, $4 \mathrm{M}$ urea were employed. Using these conditions, a purification procedure for nebulin was developed. The purified nebulin was confirmed to bind actin filaments and to form loose bundles, and therefore was able to adopt a folded state.

\section{Materials and Methods}

2.1. Protein Preparations. Actin was prepared from an acetone powder of rabbit skeletal muscle by the method of 
Spudich and Watt [14] and further purified by gel filtration through a Sephadex G-150 column equilibrated with $0.2 \mathrm{mM}$ ATP, $0.1 \mathrm{mM} \mathrm{CaCl}, 0.01 \% \mathrm{NaN}_{3}$, and $2 \mathrm{mM}$ Tris $/ \mathrm{HCl}$, $\mathrm{pH}$ 8.0. $\beta$-Actinin was purified from rabbit skeletal muscle as reported by Maruyama et al. [15], and $\alpha$-actinin was prepared from rabbit skeletal muscle by the method of Goll et al. [16]. Tropomodulin was isolated from rabbit skeletal muscle as described previously [17]. Tropomyosin was extracted from actin-extracted acetone powder of rabbit skeletal muscle and purified according to Woods [18], and troponin was prepared from rabbit skeletal muscle using the method described by Ebashi et al. [19]. The purity of the binding partners (actin, tropomyosin, troponin, tropomodulin, and $\beta$-actinin) was described in Figure 1 of the tropomodulin paper in 1999 [17], and the purity of $\alpha$ actinin was described at lane 2 in Figure 6 of the amphioxus nebulin paper in 2009 [20].

2.2. SDS-Polyacrylamide Gel Electrophoresis (PAGE) and Immunoblotting. Isolated protein samples were resolved by SDS-PAGE using $2-15 \%$ gradient polyacrylamide gels, and then transferred to nitrocellulose membranes. Membranes were treated with primary antibodies followed by secondary horseradish-labeled antibodies (Dako). Polyclonal antibodies to nebulin (PcNeb) [21], actin (A2066, Sigma-Aldrich), and connectin (Pc1200) [22] and the monoclonal antibody to connectin (Mc3B9) [23] were used.

2.3. Electron Microscopy. Negative staining was examined using $2 \%$ uranylacetate with the JEOL JEM-100S electron microscope.

2.3.1. Rotary-Shadowed Electron Microscopy. A purified nebulin solution in $50 \%$ glycerol was dropped onto a mica plate. In the case of centrifuging, the mica-taped rotor was centrifuged for $25 \mathrm{~s}$ at $12,000 \times \mathrm{g}$ as previouly reported [ $24-$ 26]. The sample was rotary-shadowed at $7.5^{\circ}$ with platinumcarbon, followed by carbon on the uncooled specimen stage of a JEOL JFD-9000 freeze fracture apparatus. The resulting specimen was observed under a JEOL 100S electron microscope.

2.4. Binding Tests by Cosedimentation. Nebulin from modified purification was dialyzed against $0.18 \mathrm{M} \mathrm{KPO}_{4}(\mathrm{pH} 7.0$ ), $0.1 \mathrm{mM} \mathrm{MgCl} 2,0.1 \mathrm{mM}$ EGTA, and $0.5 \mathrm{M}$ urea overnight and then centrifuged for 30 minutes at $56,000 \times$ g. The supernatant of nebulin was mixed with G-actin in $0.11 \mathrm{M}$ $\mathrm{KPO}_{4}, 0.27 \mathrm{mM}$ ATP, $0.1 \mathrm{mM} \mathrm{MgCl}_{2}$, and $0.31 \mathrm{M}$ urea, $\mathrm{pH} 7.0$ at $4^{\circ} \mathrm{C}$ for 20 hours. The mixture was centrifuged $\left(6,000 \times \mathrm{g}, 4^{\circ} \mathrm{C}, 30 \mathrm{~min}\right)$, and the supernatant and pellet were diluted with SDS sample buffer and subjected to SDS-PAGE.

2.5. Binding Tests by Far Western Blotting. Proteins $(0.2 \mu \mathrm{g})$ adsorbed onto a nitrocellulose membrane were first blocked with $0.2 \%$ gelatin in Tris-buffered saline and then incubated in nebulin solution $\left(0.18 \mathrm{M} \mathrm{KPO}_{4}(\mathrm{pH} 7.0), 0.1 \mathrm{mM} \mathrm{MgCl}_{2}\right.$, $0.1 \mathrm{mM}$ EGTA, and $0.5 \mathrm{M}$ urea $)(15 \mu \mathrm{g} / \mathrm{ml})$ for 20 hours at $4^{\circ} \mathrm{C}$. After washing, the membrane was treated with

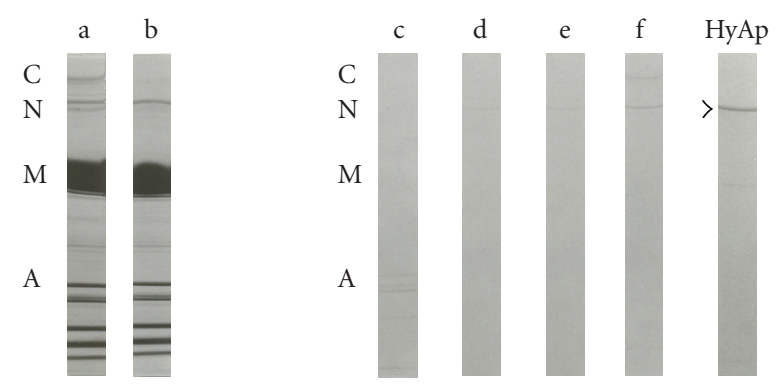

(A)

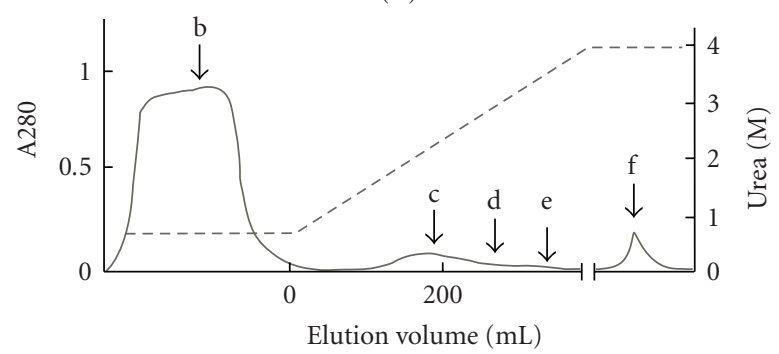

(B)

FIgURE 1: Purification of nebulin from rabbit skeletal muscle by DEAE-Toyopearl column chromatography. A muscle extract in $0.18 \mathrm{M} \mathrm{KPO}_{4}$ (pH 7.0), 0.2 mM ATP, 0.1 mM MgCl, $0.1 \mathrm{mM}$ EGTA, and $1 \mathrm{M}$ urea was loaded to DEAE-Toyopearl column and linear urea gradient up to $4 \mathrm{M}$ was applied. (A) SDS-PAGE patterns of each fraction of (B), (B) Elution pattern. a, applied sample; b, flowthrough fraction; $c-e$, eluted fraction; $f$, overnight fraction with $4 \mathrm{M}$ urea; HyAp, purified nebulin using hydroxylapatite column chromatography.

antinebulin polyclonal antibody [21] followed by secondary horseradish-labeled antibodies (Dako).

\section{Results}

3.1. Isolation of Nebulin from Rabbit Skeletal Muscle. Preliminary trials to solubilize nebulin from rabbit skeletal myofibrils proved unsuccessful under conventional salt conditions. However, with the addition of $1 \mathrm{M}$ urea, a solution containing $0.18 \mathrm{M} \mathrm{KPO}_{4}(\mathrm{pH} 7.0$ ) and $0.1 \mathrm{mM}$ EGTA could solubilize nebulin together with myosin, actin, and other muscle proteins. Purification by DEAE-Toyopearl column chromatography yielded a fraction containing nebulin and actin. However, it was very difficult to separate the two proteins. Thus, we modified the procedure to prevent actin from denaturation.

Rabbit skeletal muscle (approximately $120 \mathrm{~g}$ ) was briefly homogenized in $1 \mathrm{~L}$ of $50 \mathrm{mM} \mathrm{KCl}$ solution containing $1 \mathrm{mM} \mathrm{NaHCO} 3$ and $1 \mathrm{mM}$ EGTA and then sedimented by centrifugation for 6 minutes at 5, $000 \times \mathrm{g}$, and all procedures were carried out at $4^{\circ} \mathrm{C}$. The precipitate was suspended in the same solution and recentrifuged. This procedure was repeated four times. After washing with $5 \mathrm{mM} \mathrm{NaHCO}_{3}$, the pellet was extracted with $1 \mathrm{~L}$ of $0.2 \mathrm{M} \mathrm{NaPO}_{4}(\mathrm{pH}$ 7.0) to remove connectin and then with $0.3 \mathrm{M} \mathrm{KCl}-0.15 \mathrm{M} \mathrm{KPO}_{4}$ ( $\mathrm{pH}$ 7.0) to remove myosin. The pellet was washed three times with cold $0.1 \mathrm{mM} \mathrm{MgCl}_{2}$, and the swollen precipitate 


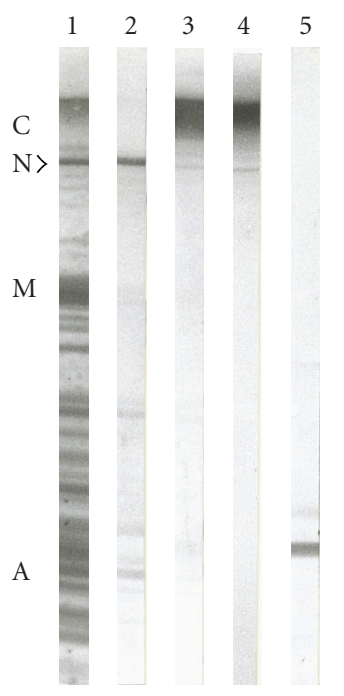

(a)

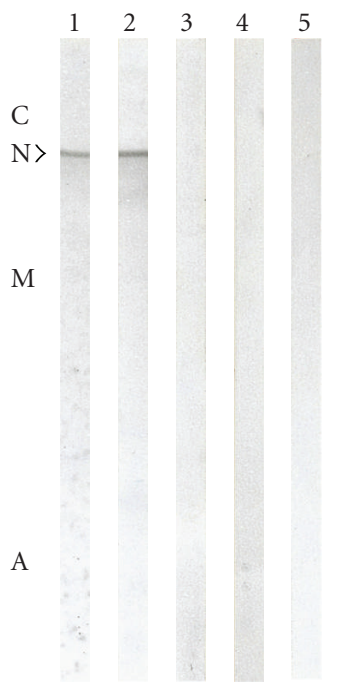

(b)
Figure 2: Immunoblot detection of purified nebulin. (a) SDS extract of whole rabbit back muscle, (b) nebulin fraction. (1) Amido black stained nitrocellulose sheets, (2) treated with PcNeb, (3) treated with Pc1200, (4) treated with Mc3B9, (5) treated with actin.

was extracted at $4^{\circ} \mathrm{C}$ for 15 minutes with an equal volume of the same precipitate in a solution with a final concentration of $0.18 \mathrm{M} \mathrm{KPO}_{4}(\mathrm{pH} 7.0), 0.2 \mathrm{mM}$ ATP, $0.1 \mathrm{mM} \mathrm{MgCl}_{2}$, and $0.1 \mathrm{mM}$ EGTA, plus $1 \mathrm{M}$ urea, which was required for nebulin extraction (Figure 1(A)-a). The sample was loaded onto the DEAE-Toyopearl $650 \mathrm{M}$ column $(1.9 \times 18 \mathrm{~cm})$ equilibrated with $0.18 \mathrm{M} \mathrm{KPO}_{4}(\mathrm{pH} 7.0), 0.1 \mathrm{M} \mathrm{MgCl}_{2}$, $0.1 \mathrm{mM}$ EGTA, and $1 \mathrm{M}$ urea, where a large amount of solubilized myosin and other proteins were not absorbed on the DEAE-Toyopearl resin (Figure 1(A)-b). Bound proteins were eluted by a gradient of 1 to $4 \mathrm{M}$ urea. Actin and nebulin were eluted at around $2 \mathrm{M}$ (Figure $1(\mathrm{~A})-\mathrm{c}$ ) and $3 \mathrm{M}$ (Figures 1(A)-d and 1(A)-e) urea, respectively. Further elution with $4 \mathrm{M}$ urea overnight $(0.02-0.03 \mathrm{mg} / \mathrm{ml})$ was more effective at eluting nebulin (Figure 1(A)-f); however, these fractions contained traces of connectin (also called titin) (Figures $1(\mathrm{~A})$-e and $1(\mathrm{~A})$-f). The eluates were dialyzed against $0.18 \mathrm{M}$ $\mathrm{KPO}_{4}(\mathrm{pH} 7.0), 0.1 \mathrm{mM} \mathrm{MgCl}, 0.1 \mathrm{mM}$ EGTA, and $2 \mathrm{M}$ urea and then centrifuged for 30 minutes at $12,000 \times \mathrm{g}$. The supernatant was loaded onto the hydroxylapatite column equilibrated with the dialysis solution. As connectin was adsorbed onto the column, nebulin alone was obtained as the flow through fraction (Figure 1(A)-HyAp), but in low yield: 0.03-0.06 mg nebulin (from $120 \mathrm{~g}$ rabbit skeletal muscle).

Immunoblotting clearly showed specific reaction to the purified nebulin fraction using anti-nebulin antibody (Figure 2(b)-2); no reaction was observed for anti-actin and anti-connectin antibodies (Figures 2(b)-3, 2(b)-4, and 2(b)-5).

3.2. Modified Purification Method for Nebulin. The protein yield was too low because our procedure was a pared preparation of nebulin. Therefore, we modified the procedure to improve the overall yield of nebulin. First, the wellwashed myofibrils were extracted with $0.18 \mathrm{M} \mathrm{KPO}_{4}(\mathrm{pH}$

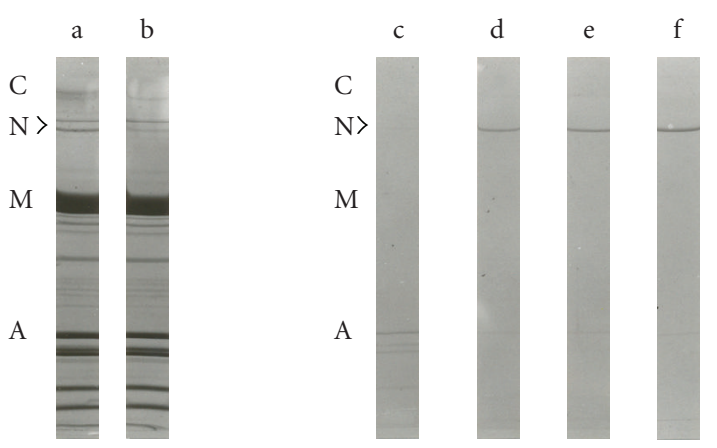

(A)

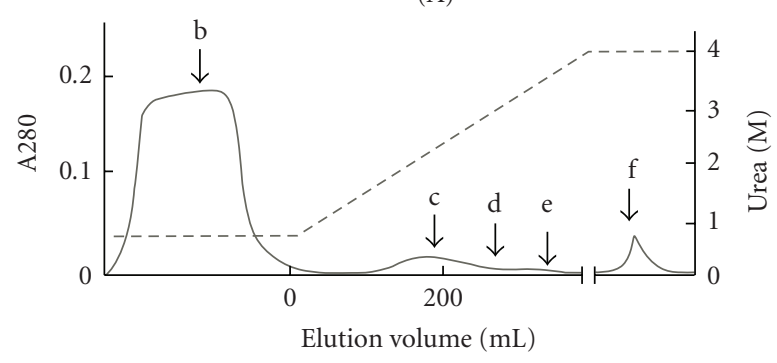

(B)

FIGURE 3: Modified purification of nebulin from rabbit skeletal muscle by DEAE-Toyopearl column chromatography. A muscle extract in $0.18 \mathrm{M} \mathrm{KPO}_{4}$ (pH 7.0), $0.1 \mathrm{mM} \mathrm{MgCl}, 0.1 \mathrm{mM}$ EGTA, and $1 \mathrm{M}$ urea was loaded to DEAE-Toyopearl column and linear urea gradient up to $4 \mathrm{M}$ was applied. (A) SDS-PAGE patterns of each fraction of (B), (B) Elution pattern. a, applied sample; b, flowthrough fraction; $\mathrm{c}-\mathrm{e}$, eluted fraction; $\mathrm{f}$, overnight fraction with $4 \mathrm{M}$ urea.

7.0), $0.1 \mathrm{mM} \mathrm{MgCl} 2,0.1 \mathrm{mM}$ EGTA, and $1 \mathrm{M}$ urea without the addition of ATP, which resulted in a smaller amount of extracted myosin (Figure 3(A)-b) than for extraction in the presence of ATP (Figure 1(A)-b). Consequently, nebulin was extracted to a larger extent under the same DEAEToyopearl column chromatography conditions described above. Overnight reelution with $4 \mathrm{M}$ urea thus resulted in a nebulin solution of $1 \sim 1.5 \mathrm{mg} / \mathrm{ml}$, although it was slightly contaminated with actin (Figure 3-f)).

3.3. Electron Microscopy of Isolated Nebulin. Rotary shadowing was applied to purified nebulin samples for visualization of its electron microscopic structure. The nebulin fraction contaminated with traces of connectin (prior to hydroxylapatite chromatography, Figures $1(\mathrm{~A})$-e and $1(\mathrm{~A})$ f) showed a number of particles and few entangled filaments (data not shown). Upon further purification of nebulin, the entangled filaments disappeared leaving only small particles (Figure 4), indicating that the filaments were contaminated with connectin molecules, as has been reported previously [25].

The remaining particles having approximately $20-\mathrm{nm}$ in diameter were regarded to be nebulin molecules (Figure 4). The particles had an irregular shape and formed a compact bundle of actin filaments, suggesting a densely aggregated form of the long nebulin molecule. Further application of centrifugal forces to extend these filamentous molecules as 


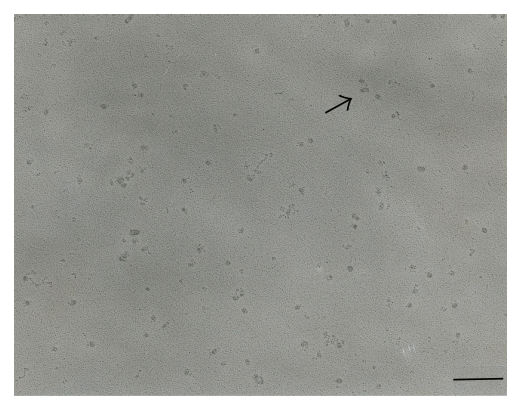

(a)

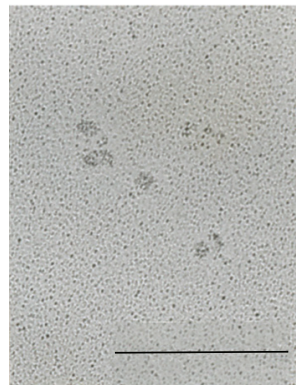

(b)
FIGURE 4: Rotary shadowed images of purified nebulin from rabbit back muscle. (a) typical images of nebulin molecules. (b), enlarged images of arrow at (a). Bar, $200 \mathrm{~nm}$.

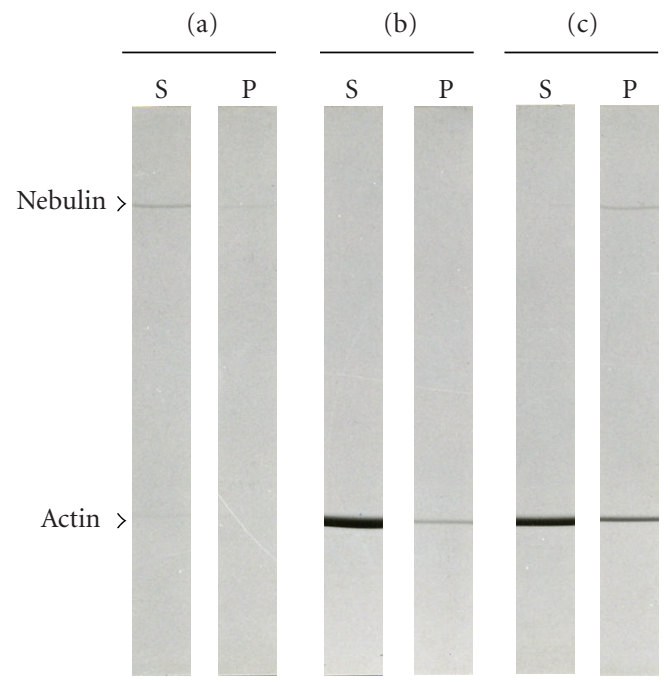

Figure 5: Binding of purified nebulin and F-actin examined by cosedimentation. (a) nebulin only, (b) actin only, (c) nebulin + actin. (S) supernatant after centrifugation, (P) pellet after centrifugation. Nebulin and actin were mixed in $0.11 \mathrm{M} \mathrm{KPO}_{4}, 0.31 \mathrm{M}$ urea, $0.27 \mathrm{mM}$ ATP, and $0.1 \mathrm{mM} \mathrm{MgCl}_{2}, \mathrm{pH}$ 7.0. Electrophoresis was performed on a $2-15 \%$ polyacrylamide gel.

previously performed for connectin [24-26] failed to change the particle shape of nebulin.

3.4. Interaction of Actin with Purified Nebulin. Purified nebulin was clarified by ultracentrifugation $(30 \mathrm{~min}, 56,000 \times$ g) and then mixed with G-actin. To polymerize G-actin, the solution conditions were changed to $0.11 \mathrm{M} \mathrm{KPO}_{4}(\mathrm{pH}$ 7.0), $0.31 \mathrm{M}$ urea, $0.27 \mathrm{mM}$ ATP and $0.1 \mathrm{mM} \mathrm{MgCl}_{2}$. The concentration of G-actin and nebulin was $0.2 \mathrm{mg} / \mathrm{ml}$ and $19 \mu \mathrm{g} / \mathrm{ml}$, respectively, giving a $1: 188$ molar ratio of nebulin to actin. After 20 hours incubation at $4^{\circ} \mathrm{C}$, the samples were centrifuged for 30 minutes at $6,000 \times \mathrm{g}$. As shown in Figure 5, nebulin coprecipitated with actin whereas nebulin alone did not precipitate; a small amount of actin alone was also precipitated. Thus, the large bundles of actin filaments were formed in the presence of nebulin, which was verified by negative-stained electron micrographs (Figure 6).
These bundles were $30 \sim 50 \mu \mathrm{m}$ long and $0.05-0.13 \mu \mathrm{m}$ wide, containing 10- to $20-\mathrm{nm}$-diameter patches thought to be the nebulin particles.

\subsection{Binding of Thin Filament Proteins to Purified Nebulin.} The interaction of nebulin with several actin-binding proteins was examined by far western blot analysis. As shown in Figure 7, $\alpha$-actinin and F-actin strongly bound to nebulin. $\beta$ Actinin and tropomodulin weakly bound to nebulin, while troponin and tropomyosin showed no binding ability.

\section{Discussion}

Since nebulin is very insoluble in salt, until now it could only be extracted and purified in the presence of a detergent such as SDS [5]. In this study, however, we were able to extract nebulin from the skeletal muscle of a rabbit by adding $1 \mathrm{M}$ urea to $0.18 \mathrm{M} \mathrm{KPO}_{4}(\mathrm{pH} 7.0)$. However, when we removed all of the urea from this nebulin crude extract solution by dialysis, the solubility of the nebulin deteriorated remarkably, and most of it precipitated. Therefore, in order to prevent nebulin from interacting with other proteins and decreasing its solubility, we purified it by column chromatography in the presence of urea.

We also tried eluting the nebulin from an ion-exchange column chromatography in a salt concentration gradient, but we were unable to remove the contaminants. We therefore, used a urea concentration gradient for elution. After the elution in the urea concentration gradient was complete, we let the nebulin stand for 12 hours and eluted it again. Consequently, we obtained a high-concentration fraction of nebulin. The reason we were able to obtain this highconcentration fraction of nebulin after the second elution, not after the first elution, might be that the nebulin needed time to dissociate from the resin in the column chromatography since the urea with which the nebulin was eluted was at a concentration of $4 \mathrm{M}$, the minimum concentration necessary for the elution. In this study, we were unable to perform a circular dichroism spectra measurement of the purified nebulin because its content was too low. However, the results of electron microscopic observation and the fact that nebulin could bind to actin suggested that the purified nebulin corresponded at least to a significant extent to a folded state.

Since nebulin is known to exist along thin filaments in skeletal muscle sarcomeres, it is thought to have a filamentous molecular shape. However, observation of isolated nebulin by the rotary shadowing method revealed that its molecular shape was not filamentous but a lump with about $20 \mathrm{~nm}$ in diameter (Figure 4). Both in the nebulin fraction obtained in the low urea concentration and the nebulin solution whose urea concentration was reduced by dialysis, the shape of nebulin was same as the nebulin eluted in $4 \mathrm{M}$ urea. Even when the nebulin was dialyzed in $\mathrm{pH} 9.0$ solution because the pI of nebulin is 9.3 [1], we did not see any change in this shape. Furthermore, even though we tried elongating the nebulin molecules by centrifuging them just like connectin, we were unable to see any change in molecular 


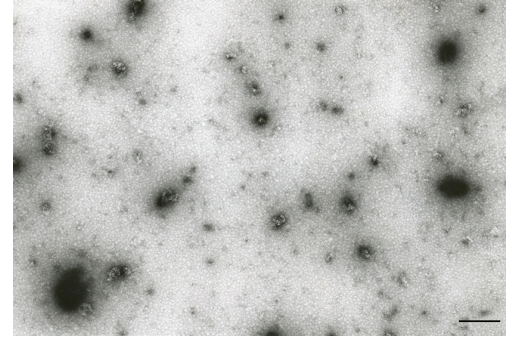

(a)

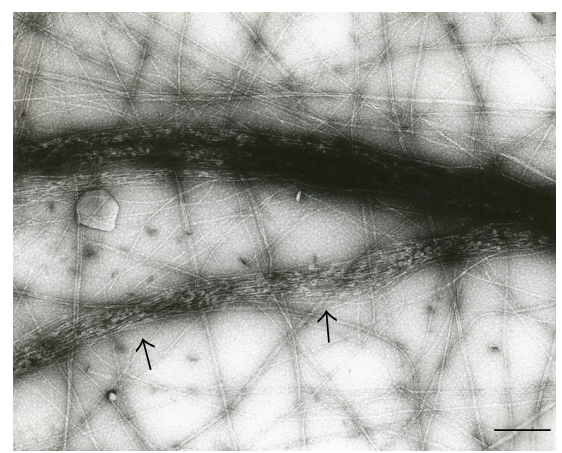

(c)

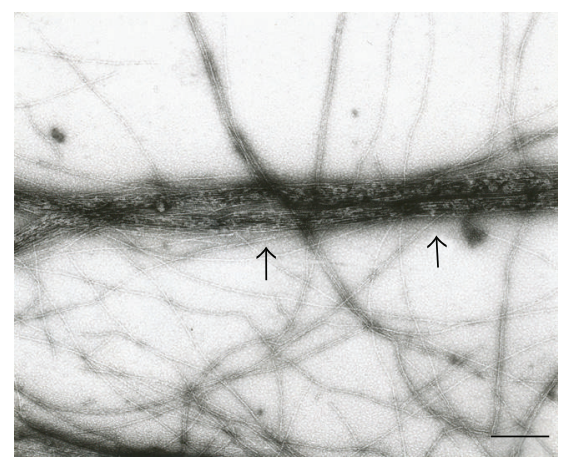

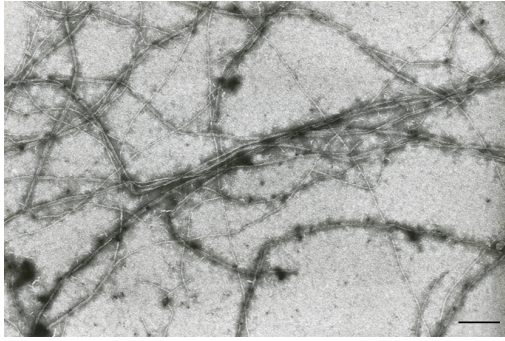

(b)
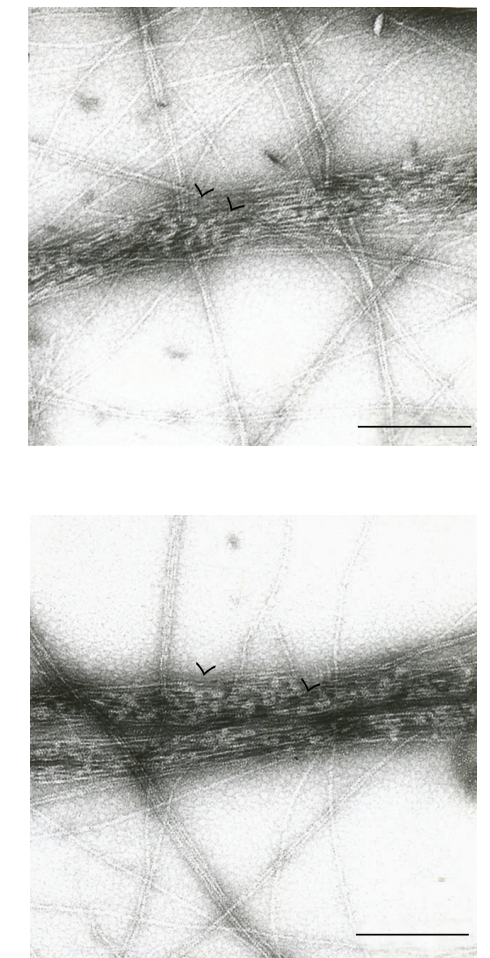

(d)

FIGURE 6: Negative-stained electron micrographs of actin filaments under the influence of purification nebulin. (a) nebulin only, $19 \mu \mathrm{g} / \mathrm{ml}$; (b) actin only, $0.2 \mathrm{mg} / \mathrm{ml}$; (c and d) actin $(0.2 \mathrm{mg} / \mathrm{ml})$ was mixed with nebulin $(19 \mu \mathrm{g} / \mathrm{ml})$ in $0.11 \mathrm{M} \mathrm{KPO}_{4}, 0.31 \mathrm{M}$ urea, $0.27 \mathrm{mM} \mathrm{ATP}$ and $0.1 \mathrm{mM} \mathrm{MgCl}_{2}$, pH 7.0. Bar, $200 \mathrm{~nm}$.

shape. We were also unable to elongate the lump-shaped molecules even when we used nonionic or amphoteric detergent. However, when we used the anionic detergent SDS, the lumped-shape molecules mostly disappeared, and we observed amorphous images (data not shown). These results suggest that since nebulin molecules are insoluble in salt, they become entangled after they are isolated from skeletal muscle. In contrast, the filamentous form of nebulin in skeletal muscle sarcomeres is probably maintained by their interaction with actin.

In this study, a cosedimentation assay revealed that nebulin isolated from skeletal muscle bound to actin (Figure 5). Other studies have verified by the ELISA method and by cosedimentation assays that the protein which is a part of the nebulin expressed in Escherichia coli bound to actin [6, 27$32]$, and the binding results of our study are consistent with the results of these studies.
Based on the contents of total proteins in muscle in vivo, it has been calculated that there are 2 molecules of nebulin per thin filament $[2,4]$. Furthermore, since it has been estimated from the primary structure that 1 molecule of actin corresponds to 1 module of nebulin in a thin filament $[1,3,4]$, human nebulin is calculated to interact with 185 actin molecules. We performed a cosedimentation assay of nebulin with actin at a ratio based on this calculation, but not all of the actin precipitated, and only part of it bound to nebulin (Figure 5). This may have been because only part of the actin-binding site of nebulin acts on actin.

On the other hand, the assay also showed that actin filaments were bundled by nebulin (Figure 6(c)). In 1998, Gonsior et al. reported that actin filament bundles were formed by the mixing of F-actin and proteins of 6 nebulin modules expressed in E. coli [31]. In our study, the bundles of actin filaments formed by the purified nebulin were 


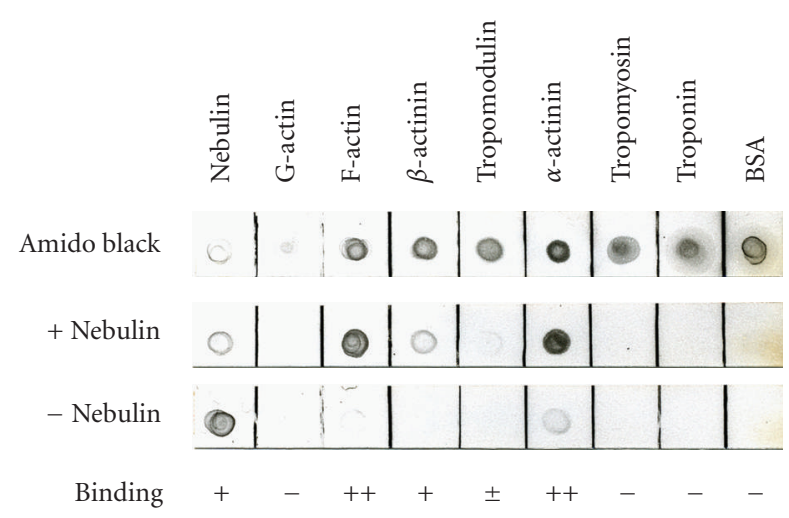

Figure 7: Binding of thin filament proteins to purified nebulin by far western blot analysis. Proteins $(0.2 \mu \mathrm{g})$ adsorbed onto a nitrocellulose membrane were incubated in nebulin reaction solution $\left(0.18 \mathrm{M} \mathrm{KPO}_{4}, 0.1 \mathrm{mM} \mathrm{MgCl}, 0.1 \mathrm{mM}\right.$ EGTA, and $0.5 \mathrm{M}$ urea, $\mathrm{pH}$ 7.0) for 20 hours at $4^{\circ} \mathrm{C}$. Amido black: amido black staining; +nebulin: incubation in nebulin $(15 \mu \mathrm{g} / \mathrm{ml})$, -nebulin: incubation in reaction solution alone.

morphologically similar to these bundles. The fact that the cosedimentation assay showed binding between nebulin and actin at a relatively weak centrifugal force also suggests that actin may be bundled by nebulin.

A widely accepted present day theory about the role of nebulin is the molecular ruler theory, which says that nebulin regulates the length of thin filaments. In skeletal muscle sarcomere, it has been shown that the barbed-end (fast-growing end) of thin filaments is capped by $\beta$-actinin at the $\mathrm{Z}$ line, while the pointed-end (slow-growing end) of the filaments is capped by tropomodulin (TMD) [33,34]. Erythrocyte tropomodulin (E-TMD) and skeletal muscle tropomodulin (Sk-TMD) among four tropomodulin isoforms have a function of length regulation of actin filaments. However, McElhinny et al. have revealed that Sk-TMD binds to nebulin with higher affinity than E-TMD does, and suggested that TMD and nebulin may work together as a linked mechanism to control thin filament lengths in skeletal muscle [9]. In this experiment, even though actin was polymerized in the presence of purified nebulin, the lengths of the actin filaments were not uniform. This may have been because actin-capping proteins were not added. In the future, it will be necessary to add $\beta$-actinin and Sk-TMD and investigate the interactions between purified nebulin and actin.

$\alpha$-Actinin is a major component of the Z-line and several different approaches have been used to dissect the set of interactions made by nebulin within the Z-disk lattice. In 1990, Nave et al. showed that chicken nebulin binds to $\alpha$ actinin by far western blot analysis [35], while in 1999, Moncman and Wang used an ELISA method to show that the SH3 domain of human nebulin binds to $\alpha$-actinin [36]. However, this was contradicted in 2000 by Ojima et al. who used the GST-pull down method to reveal that such binding does not occur [37]. In 2001, Bang et al. reported that the SH3 domain of human nebulin binds to the newly discovered protein myopalladin, and that the orientation of nebulin to the Z-line is maintained through the binding of myopalladin to $\alpha$-actinin [12]. Thus, regarding binding between $\alpha$-actinin and nebulin, the contradict results have been reported. In our study, the results of far-western blot analysis showed that purified nebulin binds directly to $\alpha$ actinin. Since the C-terminal region of nebulin localizes at the Z-line in sarcomere, nebulin and $\alpha$-actinin may bind directly and the $\mathrm{C}$-terminal region of nebulin is probably linked to the Z-line.

The N-terminal region of nebulin is known to bind to TMD which is a pointed end actin-capping protein [9], and the C-terminal region of nebulin is known to bind to $\beta$ actinin, which is a barbed end actin-capping protein. Our study showed that purified nebulin also bound to both TMD and $\beta$-actinin even though the reaction was weak (Figure 7). The reason that this binding is weak may be that the binding region of the nebulin molecule is at its terminus and is only a small portion of the entire molecule. Our study and others have shown that nebulin binds to actin capping proteins on both ends of the actin filaments. This result supports the idea that nebulin regulates the length of actin filaments.

Because nebulin is extremely insoluble in salt, biochemical analysis has not yet made progress. The novel purification method described here is likely to support future functional studies on nebulin since it provides a full-length source of nebulin peptide that allows to further study its role in thin filament assembly and its potential ruler functions.

\section{Conclusion}

In this study, nebulin was solubilized by $0.18 \mathrm{M} \mathrm{KPO}_{4}(\mathrm{pH}$ 7.0) solution containing $1 \mathrm{M}$ urea and purified by DEAEToyopearl column chromatography via $4 \mathrm{M}$ urea elution. The nebulin molecule took on a lump-like molecular shape, and we were unable to observe any molecular-ruler function. However, since the nebulin purified by the present method bound to actin and actin-capping proteins $(\beta$-actinin and tropomodulin), this nebulin may have retained some of its in vivo functions. In the future, it may become important to perform more detailed investigations of nebulin's biochemical characteristics, such as whether isolated nebulin retains its high-order structure. It may also become important to keep the concentration of urea low when purifying nebulin.

\section{Acknowledgment}

This work was supported by KAKENHI (Grants-in-Aid for Scientific Research) from the Ministry of Education, Culture, Sports, Science and Technology of Japan to Sumiko Kimura.

\section{References}

[1] S. Labeit and B. Kolmerer, "The complete primary structure of human nebulin and its correlation to muscle structure," Journal of Molecular Biology, vol. 248, no. 2, pp. 308-315, 1995.

[2] K. Wang and J. Wright, "Architecture of the sarcomere matrix of skeletal muscle: immunoelectron microscopic evidence that suggests a set of parallel inextensible nebulin filaments anchored at the Z line," The Journal of Cell Biology, vol. 107, no. 6, part 1, pp. 2199-2212, 1988. 
[3] M. Kruger, J. Wright, and K. Wang, "Nebulin as a length regulator of thin filaments of vertebrate skeletal muscles: correlation of thin filament length, nebulin size, and epitope profile," The Journal of Cell Biology, vol. 115, no. 1, pp. 97-107, 1991.

[4] S. Labeit, T. Gibson, A. Lakey, et al., "Evidence that nebulin is a protein-ruler in muscle thin filaments," FEBS Letters, vol. 282, no. 2, pp. 313-316, 1991.

[5] K. Wang, "Purification of titin and nebulin," Methods in Enzymology B, vol. 85, pp. 264-274, 1982.

[6] M. J. Chen, C.-L. Shih, and K. Wang, "Nebulin as an actin zipper. A two-module nebulin fragment promotes actin nucleation and stabilizes actin filamen," The Journal of Biological Chemistry, vol. 268, no. 27, pp. 20327-20334, 1993.

[7] N. Lukoyanova, M. S. VanLoock, A. Orlova, V. E. Galkin, K. Wang, and E. H. Egelman, "Each actin subunit has three nebulin binding sites: implications for steric blocking," Current Biology, vol. 12, no. 5, pp. 383-388, 2002.

[8] J.-P. Jin and K. Wang, "Cloning, expression, and protein interaction of human nebulin fragments composed of varying numbers of sequence modules," The Journal of Biological Chemistry, vol. 266, no. 31, pp. 21215-21223, 1991.

[9] A. S. McElhinny, B. Kolmerer, V. M. Fowler, S. Labeit, and C. C. Gregorio, "The N-terminal end of nebulin interacts with tropomodulin at the pointed ends of the thin filaments," The Journal of Biological Chemistry, vol. 276, no. 1, pp. 583-592, 2001.

[10] M.-L. Bang, C. Gregorio, and S. Labeit, "Molecular dissection of the interaction of desmin with the C-terminal region of nebulin," Journal of Structural Biology, vol. 137, no. 1-2, pp. 119-127, 2002.

[11] C. C. Witt, C. Burkart, D. Labeit, et al., "Nebulin regulates thin filament length, contractility, and Z-disk structure in vivo," EMBO Journal, vol. 25, no. 16, pp. 3843-3855, 2006.

[12] M.-L. Bang, R. E. Mudry, A. S. McElhinny, et al., "Myopalladin, a novel 145-kilodalton sarcomeric protein with multiple roles in Z-disc and I-band protein assemblies," The Journal of Cell Biology, vol. 153, no. 2, pp. 413-427, 2001.

[13] V. K. Yadavalli, J. G. Forbes, and K. Wang, "Nanomechanics of full-length nebulin: an elastic strain gauge in the skeletal muscle sarcomere," Langmuir, vol. 25, no. 13, pp. 7496-7505, 2009.

[14] J. A. Spudich and S. Watt, "The regulation of rabbit skeletal muscle contraction. I. Biochemical studies of the interaction of the tropomyosin-troponin complex with actin and the proteolytic fragments of myosin," The Journal of Biological Chemistry, vol. 246, no. 15, pp. 4866-4871, 1971.

[15] K. Maruyama, H. Kurokawa, M. Oosawa, et al., " $\beta$-Actinin is equivalent to Cap Z protein," The Journal of Biological Chemistry, vol. 265, no. 15, pp. 8712-8715, 1990.

[16] D. E. Goll, A. Suzuki, J. Temple, and G. R. Holmes, "Studies on purified $\alpha$-actinin. I. Effect of temperature and tropomyosin on the $\alpha$-actinin/F-actin interaction," Journal of Molecular Biology, vol. 67, no. 3, pp. 469-472, 1972.

[17] S. Kimura, A. Ichikawa, J. Ishizuka, S. Ohkouchi, T. Kake, and K. Maruyama, "Tropomodulin isolated from rabbit skeletal muscle inhibits filament formation of actin in the presence of tropomyosin and troponin," European Journal of Biochemistry, vol. 263, no. 2, pp. 396-401, 1999.

[18] E. F. Woods, "Peptide chains of tropomyosin," Nature, vol. 207, no. 992, pp. 82-83, 1965.

[19] S. Ebashi, T. Wakabayashi, and F. Ebashi, "Troponin and its components," Journal of Biochemistry, vol. 69, no. 2, pp. 441$445,1971$.
[20] A. Hanashima, K. Kubokawa, and S. Kimura, "Characterization of amphioxus nebulin and its similarity to human nebulin," Journal of Experimental Biology, vol. 212, no. 5, pp. 668-672, 2009.

[21] H. Sugita, I. Nonaka, Y. Itoh, et al., "Is nebulin the product of Duchenne muscular dystrophy gene?" Proceedings of Japan Academy B, vol. 63, no. 5, pp. 107-110, 1987.

[22] S. Kimura, T. Matsuura, S. Ohtsuka, Y. Nakauchi, A. Matsuno, and K. Maruyama, "Characterization and localization of $\alpha$ connectin (titin 1) an elastic protein isolated from rabbit skeletal muscle," Journal of Muscle Research and Cell Motility, vol. 13, no. 1, pp. 39-47, 1992.

[23] Y. Itoh, T. Suzuki, S. Kimura, et al., "Extensible and lessextensible domains of connectin filaments in stretched vertebrate skeletal muscle sarcomeres as detected by immunofluorescence and immunoelectron microscopy using monoclonal antibodies," Journal of Biochemistry, vol. 104, no. 4, pp. 504$508,1988$.

[24] R. Nave, D. O. Furst, and K. Weber, "Visualization of the polarity of isolated titin molecules: a single globular head on a long thin rod as the M band anchoring domain?" The Journal of Cell Biology, vol. 109, no. 5, pp. 2177-2187, 1989.

[25] M. Sonoda, S. Kimura, H. Moriya, Y. Shimada, and K. Maruyama, "Molecular shape of $\alpha$-connectin, an elastic filamentous protein of skeletal muscle," Proceedings of Japan Academy B, vol. 66, pp. 213-216, 1990.

[26] J. Suzuki, S. Kimura, and K. Maruyama, "Electron microscopic filament lengths of connectin and its fragments," Journal of Biochemistry, vol. 116, no. 2, pp. 406-410, 1994.

[27] J.-P. Jin and K. Wang, "Nebulin as a giant actin-binding template protein in skeletal muscle sarcomere. Interaction of actin and cloned human nebulin fragments," FEBS Letters, vol. 281, no. 1-2, pp. 93-96, 1991.

[28] M. J. G. Chen and K. Wang, "Conformational studies of a two-module fragment of nebulin and implications for actin association," Archives of Biochemistry and Biophysics, vol. 310, no. 2, pp. 310-317, 1994.

[29] M. Pfuhl, S. J. Winder, and A. Pastore, "Nebulin, a helical actin binding protein,” EMBO Journal, vol. 13, no. 8, pp. 1782-1789, 1994.

[30] M. Pfuhl, S. J. Winder, M. A. C. Morelli, S. Labeit, and A. Pastore, "Correlation between conformational and binding properties of nebulin repeats," Journal of Molecular Biology, vol. 257, no. 2, pp. 367-384, 1996.

[31] S. M. Gonsior, M. Gautel, and H. Hinssen, "A six-module human nebulin fragment bundles actin filaments and induces actin polymerization," Journal of Muscle Research and Cell Motility, vol. 19, no. 3, pp. 225-235, 1998.

[32] J. Q. Zhang, A. Weisberg, and R. Horowits, "Expression and purification of large nebulin fragments and their interaction with actin," Biophysical Journal, vol. 74, no. 1, pp. 349-359, 1998.

[33] D. A. Schafer and J. A. Cooper, "Control of actin assembly at filament ends," Annual Review of Cell and Developmental Biology, vol. 11, pp. 497-518, 1995.

[34] R. S. Fischer and V. M. Fowler, "Tropomodulins: life at the slow end," Trends in Cell Biology, vol. 13, no. 11, pp. 593-601, 2003.

[35] R. Nave, D. O. Furst, and K. Weber, "Interaction of alphaactinin and nebulin in vitro. Support for the existence of a fourth filament system in skeletal muscle," FEBS Letters, vol. 269, no. 1, pp. 163-166, 1990. 
[36] C. L. Moncman and K. Wang, "Functional dissection of nebulette demonstrates actin binding of nebulin-like repeats and Z-line targeting of SH3 and linker domains," Cell Motility and the Cytoskeleton, vol. 44, no. 1, pp. 1-22, 1999.

[37] K. Ojima, Z. X. Lin, M.-L. Bang, et al., "Distinct families of Z-line targeting modules in the $\mathrm{COOH}$-terminal region of nebulin," The Journal of Cell Biology, vol. 150, no. 3, pp. 553566, 2000. 

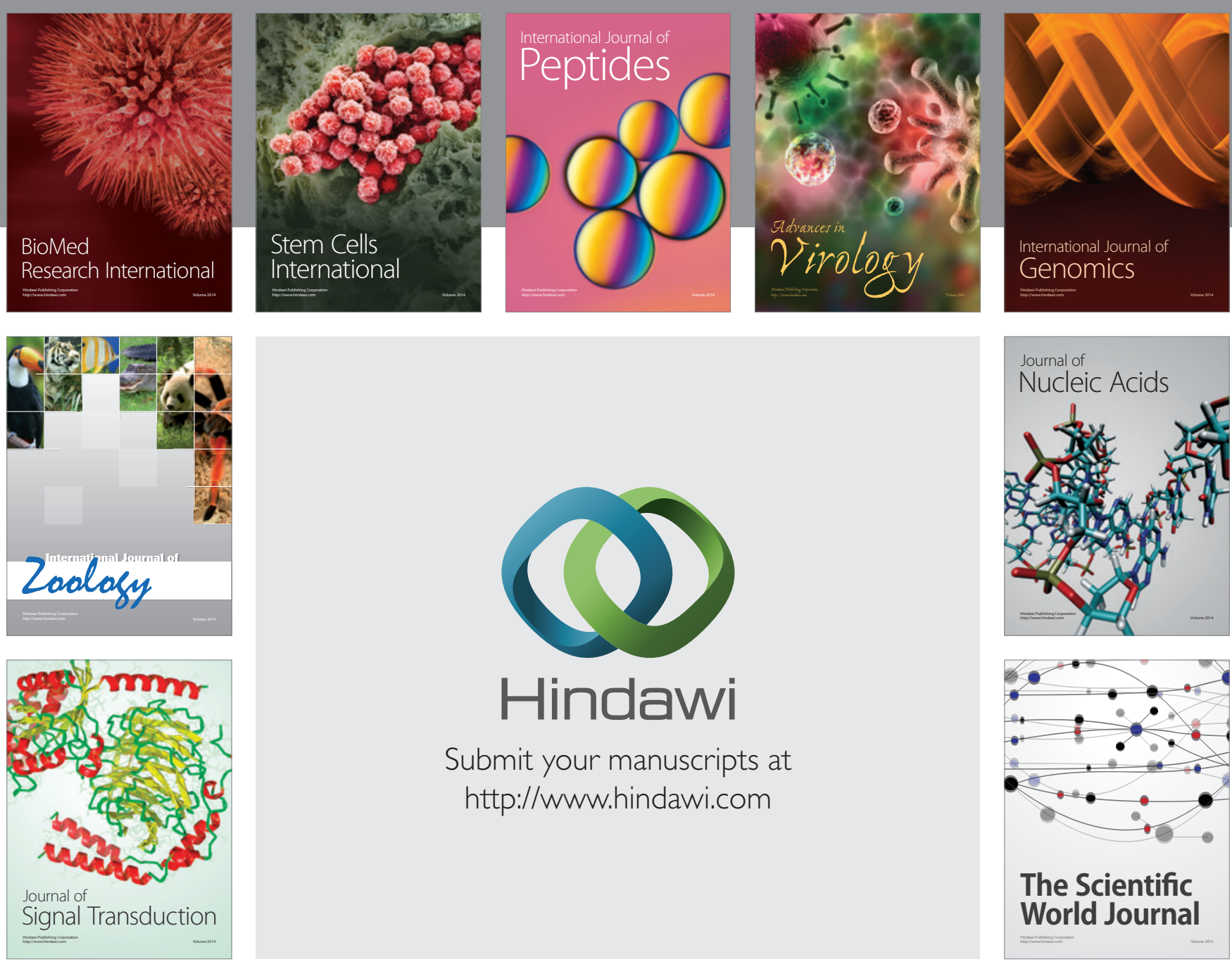

Submit your manuscripts at

http://www.hindawi.com
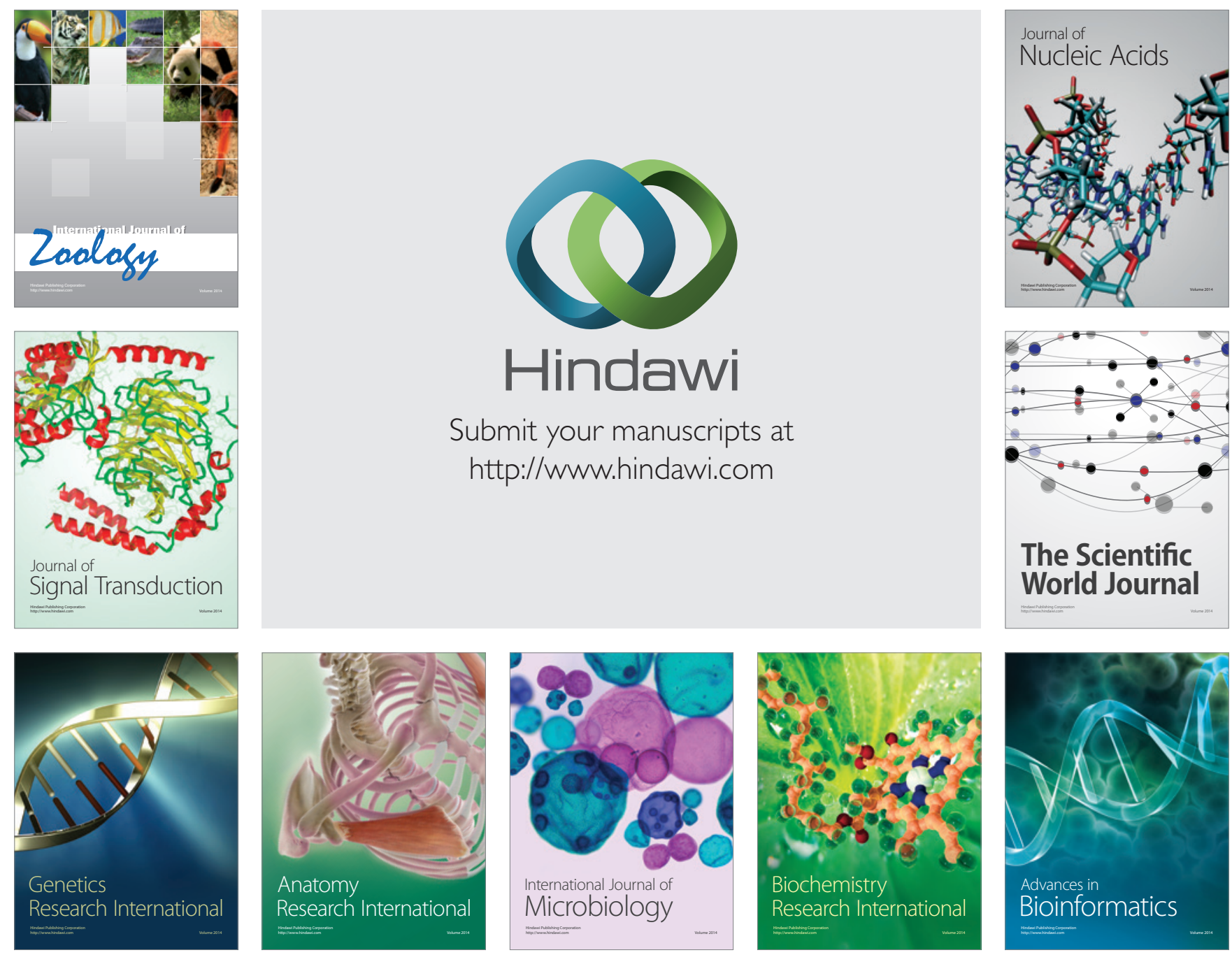

The Scientific World Journal
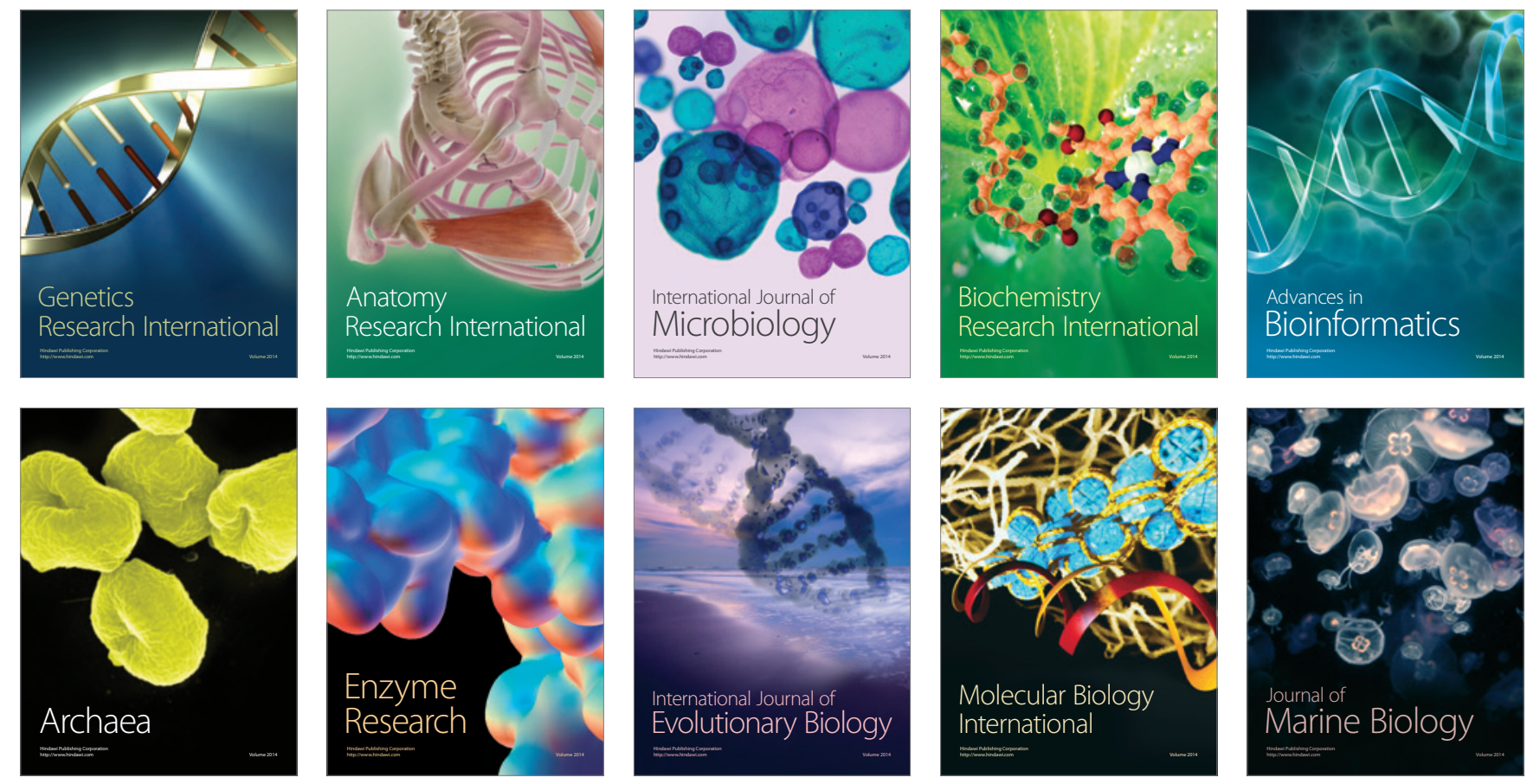